\title{
Rucaparib in relapsed, platinum-sensitive high-grade ovarian carcinoma (ARIEL2 Part 1): an international, multicentre, open-label, phase 2 trial
}

\author{
Elizabeth M Swisher*, Kevin K Lin*, Amit M Oza, Clare L Scott, Heidi Giordano, James Sun, \\ Gottfried E Konecny, Robert L Coleman, Anna V Tinker, David M O’Malley, Rebecca S \\ Kristeleit, Ling Ma, Katherine M Bell-McGuinn, James D Brenton, Janiel M Cragun, Ana Oaknin, \\ Isabelle Ray-Coquard, Maria I Harrell, Elaina Mann, Scott H Kaufmann, Anne Floquet, \\ Alexandra Leary, Thomas C Harding, Sandra Goble, Lara Maloney, Jeff Isaacson, Andrew R \\ Allen, Lindsey Rolfe, Roman Yelensky, Mitch Raponi, Iain A McNeish*
}

University of Washington, 1959 NE Pacific St, Seattle, WA, 98195 USA (Prof E M Swisher MD, M I Harrell PhD); Clovis Oncology, Inc., 5500 Flatiron Parkway, Boulder, CO, 80301, USA (K K Lin PhD, H Giordano MA, E Mann MS, T C Harding PhD, S Goble MS, L Maloney BA, J Isaacson PhD, A R Allen MD, L Rolfe MBChB, M Raponi PhD); Princess Margaret Cancer Centre, University Health Network, University Avenue, Toronto, ON, M5G 2M9, Canada (Prof A M Oza MD); Royal Melbourne Hospital, 300 Grattan St, Melbourne, Victoria 3050, Australia (C L Scott PhD); Foundation Medicine, Inc., 150 Second St, Cambridge, MA, 02141, USA (J Sun $\mathrm{PhD}, \mathrm{R}$ Yelensky PhD); University of California Los Angeles, 2825 Santa Monica Blvd, Suite 200, Santa Monica, CA 90404, USA (G E Konecny MD); The University of Texas MD Anderson Cancer Center, 1155 Herman Pressler Dr, CPB6.3590, Houston, TX 77030, USA (Prof R L Coleman MD); British Columbia Cancer Agency, $600 \mathrm{~W} \mathrm{10^{ \text {th } }}$ Ave, Vancouver, BC V5Z 4E6, Canada (A V Tinker MD); The Ohio State University, James Cancer Center, M210 Starling Loving, $320 \mathrm{~W} 10^{\text {th }}$ Ave, Columbus, OH 43210, USA (D M O’Malley MD); University College London, Cancer Institute, Paul O'Gorman Building, Huntley St, London, WC1E 6BT, UK (R S Kristeleit MD); Rocky Mountain Cancer Centers, 11750 W $2^{\text {nd }}$ Place, Suite 150, Lakewood, CO 80228, USA (L Ma MD); Memorial Sloan-Kettering Cancer Center, 1275 York Ave, New York, NY 10065, USA (K M Bell-McGuinn MD); Cancer Research UK Cambridge Institute, University of Cambridge, Li Ka Shing Centre, Robinson Way, Cambridge, CB2 ORE, UK (Prof J D Brenton FRCP); The University of Arizona Cancer Center, 1515 N Campbell Ave, PO Box 245024, Tucson, AZ 85724, USA (J M Cragun MD); Vall d'Hebron University Hospital, Vall d'Hebron Institute of Oncology (VHIO), Passeig de la Vall d'Hebron, 119-129, 08035, Barcelona, Spain (A Oaknin MD); GINECO, Centre Léon Bérard and University Claude Bernard, 28 rue Laennec, 69373, Lyon, France (Prof I Ray-Coquard MD); Mayo Clinic, 200 First St SW, Rochester, MN 55905, USA (Prof S H Kaufmann MD); Institut Bergonié, 229 cours de l'Argonne, 33076, Bordeaux, France (A Floquet MD); Gustave Roussy Cancer Center and INSERM U981, 114 Rue Edouard-Vaillant, 94805, Villejuif, France (A Leary MD); Institute of Cancer Sciences, University of Glasgow, Glasgow, G61 1QH, UK (Prof I A McNeish FRCP)

${ }^{*}$ These authors contributed equally

Correspondence to: Dr Elizabeth M. Swisher, Dept Ob/Gyn, University of Washington, 1959 NE Pacific St., Box 356460, Seattle, WA 98195-6460 USA; Phone: (206) 543-3669, Fax: (206) 5433915; swishere@uw.edu

Abstract: 413 words

Manuscript body: 3940 words
Tables and figures: 6

References: 40 


\section{Abstract}

Background Poly(ADP-ribose) polymerase (PARP) inhibitors have activity in ovarian carcinomas with homologous recombination deficiency (HRD). Along with BRCA1 and BRCA2 (BRCA) mutations, genomic loss of heterozygosity ( $\mathrm{LOH}$ ) may indicate HRD. To our knowledge, ARIEL2 is the first study to assess the utility of tumour genomic LOH, quantified by a nextgeneration sequencing assay, to predict response to rucaparib, an oral PARP inhibitor.

Methods In ARIEL2 Part 1, patients ( $n=204$ ) with recurrent, platinum-sensitive, high-grade ovarian carcinoma were classified into one of three predefined HRD subgroups based on tumour analysis: $B R C A$ mutant (deleterious germline or somatic), BRCA wild-type/LOH high, or $B R C A$ wild-type/LOH low. A cutoff of $\geq 14 \%$ was prespecified for $L O H$ high. HRD status was also assessed by additional mutational and methylation analyses. Patients initiated rucaparib $600 \mathrm{mg}$ twice daily. The primary endpoint was progression-free survival. Secondary endpoints included response rate, response duration, and safety. All patients treated with at least one dose of rucaparib were included in the primary and secondary endpoint analyses. This trial is registered with ClinicalTrials.gov, number NCT01891344. Enrolment into ARIEL2 Part 1 is complete. At the data cutoff date (18 January 2016), 28 patients remained on study.

Findings Risk of progression during rucaparib treatment was significantly reduced in the $B R C A$ mutant (hazard ratio $[\mathrm{HR}], 0 \cdot 27 ; 95 \%$ confidence interval $[\mathrm{Cl}], 0 \cdot 16-0 \cdot 44$ ) and $B R C A-$ wildtype/LOH-high (HR, 0.62; 95\% Cl, 0.42-0.90) subgroups versus the BRCA-wild-type/LOH-low subgroup. The most common treatment-emergent adverse events of any grade were nausea (163/204; 79.9\%), asthenia/fatigue (159/204; 77.9\%), constipation (94/204; 46.1\%), and vomiting $(89 / 204 ; 43.6 \%)$. The most frequent grade 3 or higher treatment-emergent adverse event was anaemia/decreased haemoglobin (43/204; $21 \cdot 1 \%)$. Common serious adverse events included small intestinal obstruction (10/204; 4.9\%), malignant neoplasm progression (10/204; 
$4.9 \%)$, and anaemia (9/204; $4.4 \%)$. Two deaths were reported on study (one case each from sepsis and disease progression). No treatment-related deaths were reported.

Interpretation In patients with $B R C A$-mutant or $B R C A$-wild-type/LOH-high platinum-sensitive ovarian carcinomas, rucaparib exhibited reduced risk of progression, and a higher response rate and longer duration of response versus patients with BRCA-wild-type/LOH-low carcinomas. These results suggest that assessment of tumour LOH can identify BRCA-wild-type platinumsensitive ovarian cancers that may benefit from rucaparib. These results extend the potential utility of PARP inhibitor therapy beyond BRCA-mutant tumours.

Funding Clovis Oncology, Inc., U.S. Department of Defense Ovarian Cancer Research Program OC120506 (EMS, SHK), Stand Up To Cancer - Ovarian Cancer Research Fund Alliance - National Ovarian Cancer Coalition Dream Team Translational Research Grant (SU2C-AACR-DT16-15) (EMS, SHK), and V Foundation Translational Award (EMS). 


\section{Research in context}

\section{Evidence before this study}

To identify other clinical trials of poly(ADP-ribose) polymerase (PARP) inhibitors for treatment of ovarian cancer, we searched PubMed for articles published through 28 July 2016, using the following search term: ("PARP inhibitor" OR rucaparib OR olaparib OR niraparib OR veliparib OR talazoparib) AND (ovarian AND (cancer OR carcinoma)). Our search identified several clinical trials demonstrating antitumour activity and/or extension of progression-free survival (PFS) with PARP inhibitor monotherapy in patients with ovarian carcinoma with or without a $B R C A$ mutation. Although some of these clinical studies suggested activity in patients without a $B R C A$ mutation, no specific biomarkers were prospectively tested. Those results highlighted that there is currently no optimal method to identify $B R C A$-wild-type cancers most likely to respond to a PARP inhibitor.

\section{Added value of this study}

ARIEL2 Part 1 demonstrated that a tumour-based, next-generation sequencing homologous recombination deficiency (HRD) assay that combines BRCA mutation status and percent of genome-wide loss of heterozygosity ( $\mathrm{LOH}$ ) could identify platinum-sensitive patients without a germline $B R C A$ mutation who are most likely to respond to rucaparib treatment. Using our novel algorithm, patients with a germline or somatic $B R C A$ mutation or wild-type $B R C A$ with high $\mathrm{LOH}$ had longer PFS and a higher objective response rate with rucaparib treatment than patients with wild-type $B R C A$ and low LOH. ARIEL2 Part 1 also demonstrated that mutation and methylation status of other homologous recombination-related genes, such as $R A D 51 C$, can be associated with high genomic LOH in BRCA-wild-type tumours and with rucaparib sensitivity.

\section{Implications of all the available evidence}


PARP inhibitors have demonstrated impressive activity in patients with a germline or somatic $B R C A$ mutation; however, there were no proven predictive biomarkers of response to PARP inhibition in those with a BRCA-wild-type tumour. ARIEL2 prospectively tested a biomarker for rucaparib responsiveness and showed that quantification of genomic LOH can identify HRD and rucaparib responsiveness in $B R C A$-wild-type cancers. These results greatly extend the utility of PARP inhibitors as a treatment for cancer. Additionally, this study provides evidence that LOH analysis is more sensitive than mutational or methylation analyses in identifying responders and should be evaluated in other tumour types where HRD may be common. 


\section{Introduction}

Ovarian cancer is the fifth leading cause of cancer death in women in both the United States and European Union. ${ }^{1,2}$ Mutations in one allele of $B R C A 1$ or $B R C A 2(B R C A)$ accompanied by loss of the wild-type allele diminishes homologous recombination-mediated DNA damage repair, ${ }^{3}$ leading to loss or duplication of chromosomal regions, also known as genomic loss of heterozygosity $(\mathrm{LOH}) \cdot{ }^{4-6} \mathrm{It}$ is estimated that half of all high-grade serous ovarian carcinomas may have homologous recombination deficiency (HRD), with approximately $15 \%$ of carcinomas harbouring a germline $B R C A$ mutation, $6 \%$ a somatic $B R C A$ mutation, and $20 \%$ a mutation in, or epigenetic silencing of, another homologous recombination gene. ${ }^{7,8}$ Even without an identifiable $B R C A$ or other known homologous recombination gene mutation, many high-grade serous ovarian carcinomas display $B R C A$-like genomic signatures, ${ }^{6,9}$ which could serve as a downstream marker of HRD.

Poly(ADP-ribose) polymerase (PARP) enzymes are involved in DNA repair through activation of the base excision repair and alternative end-joining pathways and inhibition of the nonhomologous end-joining pathway. ${ }^{10,11}$ PARP inhibition in cells with HRD is hypothesised to cause an accumulation of unrepaired DNA double-strand breaks, ultimately leading to cell death. ${ }^{10-12}$ Unlike conventional chemotherapies, PARP inhibitors are selectively lethal in HRD cells. $^{10,11,13-18}$ In clinical trials, PARP inhibitors have demonstrated antitumour activity and extended progression-free survival (PFS) in patients with or without a BRCA mutation; ${ }^{19-22}$ however, the optimal method to identify $B R C A$-wild-type cancers most likely to respond to a PARP inhibitor is unknown. ${ }^{20-23}$

Rucaparib, an oral PARP inhibitor, has demonstrated efficacy and safety in a phase 1/2 study of women with relapsed, platinum-sensitive, high-grade ovarian carcinoma harbouring a germline BRCA mutation, with an objective response rate (ORR) of $66 \cdot 7 \% .{ }^{24}$ To our knowledge, ARIEL2 
is the first study to prospectively evaluate patients with ovarian carcinoma using a tumourbased, next-generation sequencing HRD assay that combines $B R C A$ mutation status and percent of genome-wide LOH in a novel algorithm to predict rucaparib sensitivity. The aim of ARIEL2 Part 1 was to identify molecular predictors of rucaparib sensitivity in patients with platinum-sensitive recurrent high-grade ovarian carcinoma, including tumours without a germline or somatic $B R C A$ mutation.

\section{Methods}

\section{Study design and participants}

ARIEL2 (NCT01891344) is an international, multicentre, two-part, phase 2, open-label study designed to assess rucaparib sensitivity in three prospectively defined subgroups (Supplementary Appendix, pp 6, 25-153). The study was approved by the institutional review board at each study site and conducted in accordance with the Declaration of Helsinki and Good Clinical Practice Guidelines of the International Conference on Harmonisation. Patients provided written informed consent before participation. Data are presented for ARIEL2 Part 1, which has completed enrolment; an extension (Part 2) of ARIEL2, added through a protocol amendment (11 May 2015), is ongoing and will be published separately.

ARIEL2 Part 1 enrolled 206 patients (204 treated) with high-grade serous or endometrioid ovarian, fallopian tube, or primary peritoneal carcinoma who had received at least one prior platinum therapy. Patients were $\geq 18$ years old, had not previously received a PARP inhibitor, had progressed 6 months or more following their most recent platinum-based treatment, had an Eastern Cooperative Oncology Group Performance Status of 0 to 1 , and had Response Evaluation Criteria In Solid Tumors version 1.1 (RECIST)-measurable disease amenable to biopsy at trial entry. Patients were ineligible if they had an active second malignancy, had central nervous system metastases, or had received anticancer therapy $\leq 14$ days prior to 
receiving their first dose of rucaparib. Formalin-fixed paraffin-embedded archival and pretreatment tumour biopsies of adequate quality were required for each patient. A complete list of inclusion and exclusion criteria is provided in the Supplementary Appendix (pp 16-17).

\section{Procedures}

Patients initiated oral rucaparib $600 \mathrm{mg}$ twice daily for continuous 28 -day cycles until disease progression or other reason for discontinuation. Supportive care (eg, antiemetics; analgesics for pain control) was permitted at the investigator's discretion. Dose reductions (in increments of $120 \mathrm{mg}$ ) were permitted if a patient experienced a grade 3 or greater adverse event. Treatment was discontinued if a dose interruption occurred for more than 14 consecutive days (longer dose interruptions were permitted with sponsor approval). Further details on dose modifications are provided in the Supplementary Appendix ( $p 2$ ).

Disease was evaluated by the investigators per RECIST using computed tomography scans at screening and after every 8 weeks during treatment (and post-treatment for patients who discontinued for any reason other than disease progression). Patients were evaluated until confirmed disease progression or other reason for discontinuation. Cancer antigen 125 (CA125) measurements were performed at screening, day 1 of each cycle, end of treatment, and when clinically indicated. Haematology, serum chemistry, and safety assessments were performed at screening, day 1 and day 15 of cycle 1 , and day 1 of subsequent cycles.

A main objective of this trial was to prospectively test molecular predictors of rucaparib sensitivity; therefore, a next-generation sequencing-based assay (Foundation Medicine, Inc., Cambridge, MA, USA ${ }^{25}$ was used to determine the percent of genomic $\mathrm{LOH}$ in archival and pretreatment biopsies. ${ }^{25,26} \mathrm{~A}$ cutoff of $14 \%$ or greater for $\mathrm{LOH}$ high was prespecified based on analysis of The Cancer Genome Atlas (TCGA) microarray and survival data for patients with ovarian carcinoma who had received platinum-based chemotherapy (Supplementary Appendix, 
pp 2-5, 7, 18). ${ }^{7}$ Patients were classified into one of three predefined HRD subgroups based on tumour analysis: $B R C A$ mutant (deleterious germline or somatic), BRCA wild-type/LOH high, or $B R C A$ wild-type/LOH low.

The tumour sequencing assay also identified mutations in homologous recombination genes other than $B R C A 1$ or $B R C A 2$ (Supplementary Appendix, pp 3, 19-20). BRCA1 and RAD51C promoter hypermethylation was assessed in tumour using methylation-sensitive polymerase chain reaction (Supplementary Appendix, p 4). ${ }^{26,27}$ Mutations detected in tumour tissue were identified as germline or somatic by analysis of genomic DNA from blood using the BROCAhomologous recombination sequencing assay (University of Washington, Seattle, WA, USA). ${ }^{28}$ For each patient, the most recently collected tumour specimen (ie, pretreatment biopsy if available, or archival tumour if not) was used to classify BRCA mutation, genomic $\mathrm{LOH}$, and methylation status (Supplementary Appendix, pp 4-5, 8).

\section{Outcomes}

The primary endpoint was PFS, which was assessed for the interval beginning with the first dose of rucaparib to disease progression or death from any cause. Secondary endpoints included ORR, duration of response, safety, and pharmacokinetics. The ORR was defined as the proportion of patients with a best response of complete or partial response (RECIST). ${ }^{29} \mathrm{~A}$ CA-125 response was defined according to Gynecological Cancer InterGroup (GCIG) criteria. ${ }^{30}$ All RECIST and CA-125 responses reported were confirmed by a second assessment after at least 4 weeks. The combined RECIST/CA-125 ORR was assessed using GCIG combined RECIST/CA-125 criteria. $^{30}$ Duration of confirmed response (complete or partial response per RECIST) was calculated from the initial date a response was observed to the first date of progressive disease. Efficacy was assessed by the investigators. Prior to study enrolment, each patient's LOH status was unknown, and investigators were blinded to the LOH analysis during 
the study. Investigators were not blinded to $B R C A$ mutation status as patients could enrol with a known germline $B R C A$ mutation, and information regarding a $B R C A$ mutation detected upon analysis of tumour tissue during the study was provided to consenting patients/investigators.

Adverse events were classified according to Medical Dictionary for Drug Regulatory Activities classification system version $18 \cdot 1^{31}$ and graded on severity according to National Cancer Institute Common Terminology Criteria for Adverse Events version 4.03. ${ }^{32}$ Rucaparib pharmacokinetics were evaluated using trough plasma concentrations (Supplementary Appendix, pp 4-5).

\section{Statistical analysis}

After reviewing data from TCGA, we estimated that $30 \%$ of patients eligible for ARIEL2 (ie, those with platinum-sensitive ovarian cancer) would be classified in the BRCA-mutant subgroup, $30 \%$ to $50 \%$ in the BRCA-wild-type/LOH-high subgroup, and $20 \%$ to $40 \%$ in the BRCA-wildtype/LOH-low subgroup. Thus, ARIEL2 was designed to enrol at least 180 patients such that any of the three possible pairwise comparisons of subgroups would contain at least 100 patients, with each of the three comparisons resulting in $80 \%$ power at a two-sided $10 \%$ significance level to detect a difference in PFS distributions (assuming the hazard ratio [HR] between two subgroups was 0.50 ). The number of patients with a known deleterious germline $B R C A$ mutation was capped $(n=15)$. Patients who were in the screening process when the target enrolment of 180 patients was reached were allowed to complete screening and enrol into the study if eligible. All efficacy and safety analyses were performed using the safety population, which included all patients who were treated with at least one dose of rucaparib. Progression-free survival was analysed using Kaplan-Meier methodology and a Cox proportional hazard model (two-sided test at the 5\% significance level; 95\% confidence interval $[\mathrm{CI}])$ for the $B R C A$-mutant and $B R C A$-wild-type/LOH-high subgroups compared with the $B R C A-$ 
wild-type/LOH-low subgroup (Supplementary Appendix, pp 4-5, 15). Patients without documented progression were censored as of their last tumour assessment. Duration of response was analysed using Kaplan-Meier methodology, with log-rank test used to compare the distribution between subgroups. Patients with an ongoing response were censored as of their last postbaseline scan. Objective response rates were presented as percentages with $95 \%$ Cls using Clopper-Pearson methodology and analysed between subgroups using a Chi-square test of proportions. A post hoc analysis of the best percentage change in the sum of all target lesions by RECIST compared with baseline was also performed. Statistical analyses of PFS, duration of response, ORR, best percentage change in target lesions, and safety were conducted using SAS ${ }^{\circledast}$ version 9.3. Post hoc comparison of LOH classification in archival and pretreatment biopsies was analysed using Fisher's exact test. Comparison of sensitivity to detect RECIST responders using different biomarkers was analysed using McNemar's test (Supplementary Appendix, $\mathrm{p}$ 11). Statistical analyses of comparison of LOH classification and sensitivity to detect RECIST responders were conducted using $R$ version 3.3.1.

ARIEL2 is registered with ClinicalTrials.gov, number NCT01891344.

\section{Role of the funding source}

The study was designed by the funder, Clovis Oncology, Inc., and a subgroup of investigators. Data presented herein were collected by the funder; the funder and all authors interpreted and analysed the data. Writing and editorial assistance were supported by the funder. Molecular studies were funded in part by Department of Defense Ovarian Cancer Research Program OC120506 (EMS, SHK). EMS, KKL, HG, TCH, SG, L Maloney, JI, ARA, LR, MR, and IAM had access to the raw data. The corresponding author had full access to all the data in the study and had final responsibility for the decision to submit for publication.

\section{Results}




\section{Patient characteristics}

Patients were enrolled between 30 October 2013 and 19 December 2014. At the data cutoff date (18 January 2016), 204 patients were treated with rucaparib (figure 1), with 28 patients still on study. Median duration of treatment for the 204 patients was 5.7 months (interquartile range, 84.5-307.5). Steady-state pharmacokinetics with rucaparib was achieved by cycle 1 day 15 , with a mean (standard deviation) trough plasma concentration of $2026(1147) \mathrm{ng} / \mathrm{mL}$ (Supplementary Appendix, pp 5, 9). A total of 192 treated patients were classified into one of the three predefined HRD subgroups: $B R C A$ mutant (deleterious germline or somatic $B R C A$ mutation; $\mathrm{n}=40$ ), $B R C A$ wild-type/LOH high ( $\mathrm{n}=82)$, or $B R C A$ wild-type/LOH low $(\mathrm{n}=70)$. Tumours from 12 patients were determined to be $B R C A$ wild-type, but could not be classified for LOH largely because of insufficient neoplastic nuclei. Clinical characteristics of enrolled patients are detailed in table 1. Given the enrolment cap for known BRCA mutation carriers, only $9.8 \%$ of patients $(\mathrm{n}=20)$ were confirmed to have a germline BRCA mutation (14 BRCA1 and six BRCA2) using the BROCA-homologous recombination assay. Nineteen additional patients $(9 \cdot 3 \%)$ were found to have a somatic $B R C A$ mutation (14 BRCA1 and five $B R C A 2)$ based on tumour sequencing and the BROCA-homologous recombination assay. The germline/somatic status of one BRCA1 mutation was not determined. Twenty $(9 \cdot 8 \%)$ additional patients had a somatic or germline mutation in another homologous recombination gene (Supplementary Appendix, p 20). Of 165 tumours for which methylation analyses were completed, $21(12 \cdot 7 \%) B R C A 1$ and four $(2 \cdot 4 \%) R A D 51 C$ were promoter hypermethylated. Methylation of $B R C A 1$ and $R A D 51 C$ was only observed in tumours that did not harbour a germline or somatic mutation in $B R C A$ or $R A D 51 C$ (figures 2 and 3 ).

\section{Efficacy}

The risk of disease progression while on treatment with rucaparib was significantly reduced in the $B R C A$-mutant subgroup (HR, $0.27 ; 95 \% \mathrm{Cl}, 0 \cdot 16-0 \cdot 44 ; \mathrm{p}<0 \cdot 0001)$ and in the $B R C A$-wildtype/LOH-high subgroup (HR, $0.62 ; 95 \% \mathrm{Cl}, 0.42-0.90 ; \mathrm{p}=0.011)$ compared with the $B R C A-$ 
wild-type/LOH-low subgroup (figure $2 \mathrm{~A}$ ). In the $B R C A$-mutant, $B R C A$-wild-type/LOH-high, and BRCA-wild-type/LOH-low subgroups, 24, 56, and 59 patients had disease progression or died, respectively. Median PFS following rucaparib treatment was $12 \cdot 8(95 \% \mathrm{Cl}, 9 \cdot 0-14 \cdot 7), 5 \cdot 7$ (95\% $\mathrm{Cl}, 5 \cdot 3-7 \cdot 6)$, and $5 \cdot 2(95 \% \mathrm{Cl}, 3 \cdot 6-5 \cdot 5)$ months in the three subgroups. More patients in the BRCA-mutant subgroup (50.4\%; $<<0.0001$ for $\mathrm{HR}$ ) and in the BRCA-wild-type/LOH-high subgroup (28.0\%; $\mathrm{p}=0.011$ for $\mathrm{HR}$ ) were progression free at 12 months than the $B R C A$-wildtype/LOH-low subgroup (9.6\%).

The three subgroups also differed in other outcome measures. The confirmed RECIST response rates were $80.0 \%(32 / 40 ; 95 \% \mathrm{Cl}, 64 \cdot 4-90 \cdot 9), 29 \cdot 3 \%(24 / 82 ; 95 \% \mathrm{Cl}, 19 \cdot 7-40 \cdot 4)$, and $10.0 \%(7 / 70 ; 95 \% \mathrm{Cl}, 4 \cdot 1-19 \cdot 5)$ in the BRCA-mutant, BRCA-wild-type/LOH-high, and BRCAwild-type/LOH-low subgroups (table 2) and were significantly higher in the BRCA-mutant $(\mathrm{p}<0.0001)$ and $B R C A$-wild-type/LOH-high subgroups $(\mathrm{p}=0.0033)$ than in the $B R C A$-wildtype/LOH-low subgroup. The RECIST response rates were similar in patients with a germline (85.0\%, 17/20; $95 \% \mathrm{Cl}, 62 \cdot 1-96 \cdot 8)$ or somatic $(73 \cdot 7 \%, 14 / 19 ; 95 \% \mathrm{Cl}, 48 \cdot 8-90 \cdot 9) \mathrm{BRCA}$ mutation and in patients with a $B R C A 1(79.3 \%, 23 / 29 ; 95 \% \mathrm{Cl}, 60 \cdot 3-92 \cdot 0)$ or $B R C A 2(81.8 \%$, $9 / 11 ; 95 \% \mathrm{Cl}, 48 \cdot 2-97 \cdot 7)$ mutation (table 2). Post hoc analysis of the best percentage change in the sum of all target lesions by RECIST compared with baseline is shown for each patient according to molecular subgroup in figure 3. The confirmed combined RECIST/CA-125 response rates were $85 \cdot 0 \%(34 / 40 ; 95 \% \mathrm{Cl}, 70 \cdot 2-94 \cdot 3), 43 \cdot 9 \%(36 / 82 ; 95 \% \mathrm{Cl}, 33 \cdot 0-55 \cdot 3)$, and $20.0 \%(14 / 70 ; 95 \% \mathrm{Cl}, 11.4-31 \cdot 3)$ in the BRCA-mutant, BRCA-wild-type/LOH-high, and $B R C A$-wild-type/LOH-low subgroups (table 2), and were significantly higher in the BRCAmutant $(\mathrm{p}<0.0001)$ and BRCA-wild-type/LOH-high $(\mathrm{p}=0.0018)$ subgroups than in the $B R C A-$ wild-type/LOH-low subgroup. 
Median duration of response was 9.2 months $(95 \% \mathrm{Cl}, 6 \cdot 4-12.9)$ in the $B R C A$-mutant subgroup and $10 \cdot 8$ months $(95 \% \mathrm{Cl}, 5 \cdot 7-$ not reached) in the BRCA-wild-type/LOH-high subgroup, durations which were both longer $(p=0.013$ and $p=0.022)$ than that observed for the $B R C A-$ wild-type/LOH-low subgroup (5.6 months; 95\% Cl, 4.6-8.5; Supplementary Appendix, p 10).

Both RECIST and CA-125 responses were observed in patients with a mutation in a non-BRCA homologous recombination gene (eg, $A T M, N B N, R A D 51 C$, or RAD51D) (Supplementary Appendix, p 20). Confirmed RECIST responses were also seen in patients with $B R C A 1$ methylated and $R A D 51 C$ methylated tumours (figure $2 \mathrm{~B}$ ).

Among BRCA-wild-type tumours, genomic LOH was a more sensitive predictor of response (sensitivity, $78 \%$ ) than mutation of other homologous recombination genes (sensitivity, 11\%; $\mathrm{p}<0.0001$ per McNemar's test) and methylation of BRCA1 or RAD51C (sensitivity, $48 \%$;

$\mathrm{p}<0.021$; Supplementary Appendix, p 11). However, genomic LOH was not significantly more sensitive than an analysis that combined both mutation and methylation (sensitivity, 59\%; $p=0 \cdot 13)$

\section{Archival versus pretreatment biopsies}

For 117 patients, LOH analyses were completed on paired archival and pretreatment tissue; post hoc analysis indicated that LOH classification was highly concordant $(r=0.86, p<0.0001$; Supplementary Appendix, p 12). Of 50 patients with an LOH-low archival specimen, 34.0\% $(17 / 50)$ had an LOH-high pretreatment specimen. Of the 17 patients with a change in classification from LOH low to LOH high, five had a partial response. In contrast, the reverse scenario of a change in classification from LOH high to $\mathrm{LOH}$ low between the archival and the pretreatment tissue was not observed for any patient. Methylation of $B R C A 1$ was also highly concordant in 90 paired samples ( $p<0.0001$; Supplementary Appendix, $p$ 12). Of 13 patients with BRCA1 methylation in the archival specimen, $30 \cdot 8 \%(4 / 13)$ had an unmethylated 
pretreatment sample. Only one patient had methylation in the pretreatment biopsy but not in the archival biopsy.

\section{Safety}

At least one treatment-emergent adverse event was reported in 100.0\% (204/204) of patients. Common treatment-emergent adverse events included nausea (163/204; 79.9\%) and asthenia/fatigue (159/204; 77.9\%) (table 3). The most frequent grade 3 or higher treatmentemergent adverse event was anaemia/decreased haemoglobin (43/204; $21 \cdot 1 \%)$. Treatmentemergent elevations in alanine aminotransferase (ALT), aspartate aminotransferase (AST), and blood creatinine, which were predominantly grade 1 or 2 , were also observed. One or more serious treatment-emergent adverse event was reported in $24.5 \%(50 / 204)$ of patients. Common serious adverse events included small intestinal obstruction (10/204; 4.9\%), malignant neoplasm progression (10/204; 4.9\%), and anaemia (9/204; 4.4\%) (Supplementary Appendix, p 21). Thirty-nine percent of all treated patients (80/204) required a dose reduction, most commonly for anaemia $(28 / 204 ; 13.7 \%)$ and nausea $(22 / 204 ; 10 \cdot 8 \%)$ (Supplementary Appendix, p 22). Nineteen patients (9.3\%) discontinued treatment with an adverse event as the primary reason; fatigue was the most common reason in six patients (2.9\%) (Supplementary Appendix, $p$ 23). Two deaths were reported on study (one case each because of sepsis and malignant neoplasm progression). No treatment-related deaths were reported.

\section{Discussion}

The results of ARIEL2 Part 1 demonstrate the activity of rucaparib in patients with relapsed platinum-sensitive, high-grade ovarian carcinoma. These data also highlight the ability of an HRD signature identified by an algorithm that combines the percent of tumour genomic LOH with $B R C A$ mutation status to identify patients who may benefit from rucaparib treatment. In ARIEL2 Part 1, the three groups defined by BRCA and LOH analysis had distinct outcomes. 
The BRCA-mutant subgroup had a significantly lower risk of progression $(\mathrm{HR}, 0.27)$ and a higher RECIST response rate $(80.0 \% ; p<0.0001)$ than patients with $B R C A$-wild-type/LOH-low carcinomas (10.0\%). Response rates were similar between rucaparib-treated patients with a somatic or germline $B R C A$ mutation and with a $B R C A 1$ or $B R C A 2$ mutation.

For patients with a $B R C A$-wild-type carcinoma, there was increased benefit for $\mathrm{LOH}$-high compared to those with an LOH-low carcinoma. Although the two BRCA-wild-type subgroups had a similar median PFS (5.7 and 5.2 months in LOH-high and LOH-low), the BRCA-wildtype/LOH-high subgroup had a greater chance of being progression free at any time point (HR, $0.62 ; p=0.0113$; figure $2 A)$, better confirmed RECIST response rate $(29.3 \%$ vs $10.0 \%$; $\mathrm{p}=0.0033)$, better confirmed RECIST/CA-125 response rate $(43.9 \%$ vs $20.0 \% ; \mathrm{p}=0.0018)$, and longer duration of response (median, 10.8 vs 5.6 months; $\mathrm{p}=0.0221$ ) than the $\mathrm{LOH}$-low subgroup. The median duration of response for the BRCA-wild-type/LOH-high subgroup (10.8 months; $95 \% \mathrm{Cl}, 5 \cdot 7-$ not reached) was similar to that of the BRCA-mutant subgroup (9.2 months; $95 \% \mathrm{Cl}, 6 \cdot 4-12 \cdot 9$ ), with $15.9 \%$ and $30.0 \%$ of patients still on treatment at the cutoff date, indicating the ability of the HRD assay to identify patients without a BRCA mutation who may experience a durable response with rucaparib treatment. A retrospective analysis of these data indicated that a refined cutoff of $16 \%$ or greater in the $\mathrm{LOH}$ high subgroup provided optimal discrimination of PFS, ORR, and duration of response in patients with BRCA-wild-type ovarian carcinomas. ${ }^{33}$

Comparing outcomes in ARIEL2 Part 1 to other studies investigating PARP inhibitors is difficult because of the ambiguity in how $B R C A$-wild-type cancers have been defined historically. For example, in a recent study of patients with recurrent platinum-sensitive ovarian carcinoma, median PFS was 5.7 months and the ORR was $31.8 \%$ following use of single-agent olaparib in a subgroup of 22 patients without a germline $B R C A$ mutation. However, the $B R C A$ mutation 
status of the tumour was unknown in half of the patients in that subgroup (11/22). ${ }^{34}$ Additionally, we are not aware of any studies that have prospectively investigated PFS or ORR following platinum therapy in patients with relapsed, $B R C A$-wild-type ovarian carcinoma, which makes it difficult to compare the results from ARIEL2 Part 1 to an expected response rate to platinum therapy.

Our results add to the increasing body of evidence demonstrating the potential of HRD analysis to identify patients who will benefit from PARP inhibitor treatment. Other biomarkers for HRD have been evaluated in prior studies, ${ }^{4,35,36}$ for example, through retrospective analysis of $B R C A$ mutations in ovarian carcinoma, ${ }^{21}$ or prospective identification of homozygous deletions/mutations through next-generation sequencing in prostate cancer. ${ }^{22}$ Additionally, the NOVA trial (NCT01847274) is prospectively testing an HRD-based assay in a trial of niraparib as maintenance therapy in patients with platinum-sensitive ovarian cancer. However, we believe that ARIEL2 is the only study to prospectively assess an HRD assay in ovarian cancer patients with measurable disease treated with a PARP inhibitor, thereby testing the assay as a biomarker for PARP inhibitor response. Other prospective trials in ovarian cancer are evaluating HRD assays in the maintenance setting following platinum therapy (eg, NOVA, ARIEL3).

Our results also suggest that, in platinum-sensitive ovarian carcinomas, a mutation in a homologous recombination gene other than $B R C A 1$ or $B R C A 2$ (eg, $R A D 51 C$ ) or promoter hypermethylation of $B R C A 1$ or $R A D 51 C$ can be associated with high genomic $L O H$ and with rucaparib response (figures 2 and 3; Supplementary Appendix, p 20). However, not all homologous recombination gene mutations were associated with an LOH-high genotype. Although the $\mathrm{LOH}$ analysis was more sensitive in identifying responders in BRCA-wild-type ovarian carcinomas than were either mutational or methylation analyses, $\mathrm{LOH}$ analysis was not significantly more sensitive than mutation and methylation analyses combined. The high 
correlation of genomic LOH in archival and pretreatment biopsies indicates that either source can be used to predict response to rucaparib in this patient population (Supplementary Appendix, $p$ 12). However, a subset of patients whose archival tumour samples were defined as having low genomic $\mathrm{LOH}$ had increased genomic $\mathrm{LOH}$ in matched pretreatment tumour biopsies. This corresponded to higher predictive sensitivity for recent biopsies (Supplementary Appendix, $\mathrm{p}$ 13). Interestingly, even in this platinum-sensitive patient population, loss of $B R C A 1$ methylation from archival to pretreatment biopsy was observed in $30.8 \%$ of tumours. Recent data from ovarian cancer patients with acquired chemotherapy resistance demonstrated that loss of $B R C A 1$ methylation could serve as a mechanism of therapeutic resistance.$^{37}$ Given that the HRD status within a tumour may change over time, we recommend testing the most recently collected tumour biopsy.

In ARIEL2, treatment-emergent toxicities were frequent and led to dose reductions in $39 \cdot 2 \%$ (80/204) of patients; however, only $9 \cdot 3 \%$ (19/204) of patients withdrew from the study as a result of a treatment-emergent toxicity. As with studies of other PARP inhibitors, treatmentemergent anaemia was the most common grade 3 adverse event. Anaemia was managed through transfusions and dose reductions. Alanine/aspartate aminotransferase levels increased following use of rucaparib; however, these increases were asymptomatic, reversible, and rarely associated with increased bilirubin levels. Patients with elevated ALT/AST levels were able to continue rucaparib treatment without dose reduction, and these elevations normalised over time.

Mild to moderate elevations in creatinine were also observed within the first few weeks following initiation of rucaparib treatment. Veliparib, another PARP inhibitor, has been shown to inhibit drug transporters expressed in the liver (MATE1) and kidneys (OCT2, MATE1, and MATE2K). ${ }^{38}$ Similarly, in vitro studies have shown that rucaparib inhibits MATE1 and MATE2-K 
transporters, which play a role in renal secretion of creatinine. Thus, inhibition of these transporters may be responsible for the increases in blood creatinine observed following rucaparib treatment. Based on this mechanism, elevations in serum creatinine should be evaluated in conjunction with other laboratory parameters to assess renal function.

Our study had several limitations. Although ARIEL2 Part 1 identified a biomarker that appears predictive, it remains possible that the HRD assay may only be prognostic; therefore, the predictive ability of the biomarker will need to be confirmed in the setting of a larger randomised study. Given these considerations, the refined LOH-high cutoff of $16 \%$ or greater that was identified retrospectively in ARIEL2 Part 1 is being prospectively applied in the randomised, phase 3 study ARIEL3 (NCT01968213), which is assessing PFS and overall survival with rucaparib as maintenance therapy following platinum-based chemotherapy for patients with platinum-sensitive, recurrent ovarian carcinoma. The randomised design of ARIEL3 will enable confirmation of genomic LOH as a predictive biomarker. Additionally, it is not known whether the findings in ARIEL2 Part 1 will extend to patients whose disease is resistant or refractory to platinum therapy. Hence, the HRD assay is also being prospectively tested in an extension (Part 2) of ARIEL2 that is investigating rucaparib in patients who are platinum-sensitive, -resistant, or -refractory; have received at least three but not more than four prior chemotherapies; and had a treatment-free interval of more than 6 months following first-line chemotherapy. The primary endpoint of ARIEL2 Part 2 is the ORR; PFS and overall survival are key secondary endpoints. Additional studies should assess whether the HRD assay developed in ARIEL2 predicts sensitivity to rucaparib and other PARP inhibitors in patients with other cancer types, including nonserous ovarian, gastric, pancreatic, prostate, or breast cancers..$^{9,22,39-41}$

\section{Contributors}


EMS, CLS, HG, SHK, ARA, LR, MR, and IAM were involved in the study conception.

EMS, KKL, HG, TCH, L Maloney, JI, ARA, LR, MR, and IAM were involved in the study design.

EMS, RLC, RSK, JDB, SHK, and IAM acquired funding.

EMS, KKL, and IAM were involved in the protocol development and cowrote the first draft of the manuscript.

EMS, AMO, CLS, GEK, RLC, AVT, DMO, RSK, L Ma, KMB-M, JDB, JMC, AO, IR-C, AF, AL, and IAM treated patients.

EMS, KKL, AMO, CLS, GEK, RLC, AVT, DMO, RSK, L Maloney, KMB-M, JDB, JMC, AO, IR-C, $\mathrm{MIH}, \mathrm{SHK}, \mathrm{AF}, \mathrm{AL}$, and IAM acquired data.

EMS, KKL, CLS, HG, JS, SHK, TCH, SG, L Maloney, JI, ARA, LR, RY, MR, and IAM interpreted the data.

EM contributed to sample acquisition and management.

MIH analysed data.

All authors contributed to manuscript revisions and approved the final draft for submission.

\section{Declaration of interests}

KKL, HG, EM, TCH, SG, L Maloney, JI, LR, and MR are employees of Clovis Oncology; ARA was employed at Clovis Oncology at the time of the study and owns stock. CLS's institution received in kind research support for parallel laboratory work using rucaparib. JS is an employee of and RY was an employee of Foundation Medicine, the developer of the HRD assay used in ARIEL2. RLC reports grants from AstraZeneca, Roche/Genentech, Janssen, OncoMed, Millennium, Esperance, and AbbVie. AVT has served on an advisory board for and received grants from AstraZeneca. DMO received research funding from Clovis Oncology; received 
institutional research support from Amgen, VentiRx, Regeneron, Immunogen, Array Biopharma, Janssen R\&D, Clovis Oncology, EMD Serono, Ergomed, Ajinomoto, and Genentech/Roche; and served on a steering committee or advisory boards for Amgen, AstraZeneca, Janssen, Clovis Oncology, Genentech/Roche, and Eisai. During the conduct of the study, RSK served on an advisory board for Clovis Oncology. KMB-M served on advisory boards for Clovis Oncology and AstraZeneca. JDB has been advisor for and owns stock in Inivata, has served on a speakers' bureau for AstraZeneca, has received nonfinancial support from Clovis Oncology and Aprea $A B$, and has a pending patent for a diagnostic method of relevance to the current work. AO has served on advisory boards for Roche, AstraZeneca, Pharmamar, and Clovis. IR-C has served on an advisory board for AstraZeneca, Pharmamar, and Roche. SHK has a patent for a diagnostic method of relevance to the current work. AL has served on an advisory board for Clovis, Pfizer, and Pharmamar, and reports institutional research grant support from Gamamabs and Merus. IAM has served on advisory boards for Clovis Oncology and AstraZeneca. All other authors have nothing to disclose.

\section{Acknowledgments}

The ARIEL2 study was funded by Clovis Oncology, Inc. Funding was also provided by the U.S. Department of Defense Ovarian Cancer Research Program OC120506 [EMS and SHK], a V Foundation Translational Award [EMS], and a Stand Up To Cancer - Ovarian Cancer Research Fund Alliance - National Ovarian Cancer Coalition Dream Team Translational Research Grant (Grant Number: SU2C-AACR-DT16-15) [EMS and SHK]. Stand Up to Cancer is a program of the Entertainment Industry Foundation; research grants are administered by the American Association for Cancer Research, a scientific partner of Stand Up To Cancer. Additional support was provided in part by the Ann Rife Cox Chair in Gynecology and the Judy Reis (Al Pisani) 
Ovarian Cancer Research Fund $[R L C]$ and the $\mathrm{UCH} / \mathrm{UCL}$ Biomedical Research Centre $[\mathrm{RK}]$ and the Experimental Cancer Medicine Centres at UCL [RK], Cambridge [JDB] and Glasgow [IAM].

First and foremost, we thank all of the patients and their families and caregivers for their participation in ARIEL2. In addition, we thank all of the ARIEL2 investigators (see the Supplementary Appendix, $\mathrm{p}$ 2) for their contributions to the administration and execution of the trial.

We thank Mike Bartosiewicz, Jennifer Borrow, Amanda Cha, Kathy Crankshaw, Erin Dominy, Monica Roy, Sanjay Shetty, and Simon Watkins for clinical development and operational support of the ARIEL2 study. We thank Matthew Hawryluk, Murtaza Mehdi, Vince Miller, Phil Stephens, and Scott Yerganian for scientific guidance and operational support in the development of the NGS-based HRD assay. We thank Andy Simmons and Jim Xiao for their assistance with preclinical and pharmacokinetic data relating to this manuscript. We thank Yuting He, Adrienne Johnson, and Peter Morello for assistance in manuscript preparation. Writing and editorial assistance funded by Clovis Oncology, Inc., was provided by Nathan Yardley, PhD, and Shannon Davis of Infusion Communications (Haddam, CT, USA). 


\section{References}

1 National Cancer Institute (NCI). SEER Cancer Statistics Factsheets: Ovary cancer. http://seer.cancer.gov/statfacts/html/ovary.html (accessed September 29 2015).

2 Ferlay J, Soerjomataram I, Erik M, et al. GLOBOCAN 2012 v1.0, Cancer incidence and mortality worldwide: IARC CancerBase No. 11 [Internet]. Lyon, France: International Agency for Research on Cancer. 2013 2013. http://globocan.iarc.fr/Pages/summary_table_pop_sel.aspx (accessed September 29 2015).

3 Watkins JA, Irshad S, Grigoriadis A, Tutt AN. Genomic scars as biomarkers of homologous recombination deficiency and drug response in breast and ovarian cancers. Breast Cancer Res 2014; 16: 211.

4 Abkevich V, Timms KM, Hennessy BT, et al. Patterns of genomic loss of heterozygosity predict homologous recombination repair defects in epithelial ovarian cancer. Br J Cancer 2012; 107: 1776-82.

$5 \quad$ Pedersen B, Konstantinopoulos PA, Spillman MA, De S. Copy neutral loss of heterozygosity is more frequent in older ovarian cancer patients. Genes Chromosomes Cancer 2013; 52: 794-801.

6 Marquard AM, Eklund AC, Joshi T, et al. Pan-cancer analysis of genomic scar signatures associated with homologous recombination deficiency suggests novel indications for existing cancer drugs. Biomark Res 2015; 3: 9.

7 The Cancer Genome Atlas (TCGA) Research Network. Integrated genomic analyses of ovarian carcinoma. Nature $2011 ; 474: 609-15$.

8 Pennington KP, Walsh T, Harrell MI, et al. Germline and somatic mutations in homologous recombination genes predict platinum response and survival in ovarian, fallopian tube, and peritoneal carcinomas. Clin Cancer Res 2014; 20: 764-75. 
9 Konstantinopoulos PA, Spentzos D, Karlan BY, et al. Gene expression profile of BRCAness that correlates with responsiveness to chemotherapy and with outcome in patients with epithelial ovarian cancer. J Clin Oncol 2010; 28: 3555-61.

10 De Lorenzo SB, Patel AG, Hurley RM, Kaufmann SH. The elephant and the blind men: making sense of PARP inhibitors in homologous recombination deficient tumor cells. Front Oncol 2013; 3: 228.

11 Scott CL, Swisher EM, Kaufmann SH. Poly (ADP-ribose) polymerase inhibitors: recent advances and future development. J Clin Oncol 2015; 33: 1397-406.

12 Ceccaldi R, Liu JC, Amunugama R, et al. Homologous-recombination-deficient tumours are dependent on Poltheta-mediated repair. Nature 2015; 518: 258-62.

13 Bryant HE, Schultz N, Thomas HD, et al. Specific killing of BRCA2-deficient tumours with inhibitors of poly(ADP-ribose) polymerase. Nature 2005; 434: 913-7.

14 Farmer $\mathrm{H}$, McCabe N, Lord CJ, et al. Targeting the DNA repair defect in BRCA mutant cells as a therapeutic strategy. Nature 2005; 434: 917-21.

15 McCabe N, Turner NC, Lord CJ, et al. Deficiency in the repair of DNA damage by homologous recombination and sensitivity to poly(ADP-ribose) polymerase inhibition. Cancer Res 2006; 66: 8109-15.

16 Mendes-Pereira AM, Martin SA, Brough R, et al. Synthetic lethal targeting of PTEN mutant cells with PARP inhibitors. EMBO Mol Med 2009; 1: 315-22.

17 McEllin B, Camacho CV, Mukherjee B, et al. PTEN loss compromises homologous recombination repair in astrocytes: implications for GBM therapy with temozolomide or PARP inhibitors. Cancer Res 2010; 70: 5457-64.

18 Williamson CT, Muzik H, Turhan AG, et al. ATM-deficiency sensitizes mantle cell lymphoma cells to poly(ADP-ribose) polymerase-1 inhibitors. Mol Cancer Ther 2010; 9: 347-57. 19 Fong PC, Boss DS, Yap TA, et al. Inhibition of poly(ADP-ribose) polymerase in tumors from BRCA mutation carriers. N Engl J Med 2009; 361: 123-34. 
20 Gelmon KA, Tischkowitz M, Mackay H, et al. Olaparib in patients with recurrent highgrade serous or poorly differentiated ovarian carcinoma or triple-negative breast cancer: a phase 2, multicentre, open-label, non-randomised study. Lancet Oncol 2011; 12: 852-61. 21 Ledermann J, Harter P, Gourley C, et al. Olaparib maintenance therapy in patients with platinum-sensitive relapsed serous ovarian cancer: a preplanned retrospective analysis of outcomes by BRCA status in a randomised phase 2 trial. Lancet Oncol 2014; 15: 852-61. 22 Mateo J, Carreira S, Sandhu S, et al. DNA-repair defects and olaparib in metastatic prostate cancer. N Engl J Med 2015; 373: 1697-708.

23 Lee JM, Ledermann JA, Kohn EC. PARP Inhibitors for BRCA1/2 mutation-associated and BRCA-like malignancies. Ann Oncol 2014; 25: 32-40.

24 Shapira-Frommer R, Oza AM, Domchek SM, et al. A phase 2 open-label, multicenter study of single-agent rucaparib in the treatment of patients with relapsed ovarian cancer and a deleterious BRCA mutation. Eur J Cancer 2015; 51: S545; abstract 2746.

25 Frampton GM, Fichtenholtz A, Otto GA, et al. Development and validation of a clinical cancer genomic profiling test based on massively parallel DNA sequencing. Nat Biotechnol 2013; 31: 1023-31.

26 Lin K, Sun J, Maloney L, et al. Quantification of genomic loss of heterozygosity enables prospective selection of ovarian cancer patients who may derive benefit from the PARP inhibitor rucaparib. Eur J Cancer 2015; 51: S531-2; abstract 2701.

27 Sun JX, Frampton G, Wang K, et al. A computational method for somatic versus germline variant status determination from targeted next-generation sequencing of clinical cancer specimens without a matched normal control. Cancer Res 2014; 74: abstract 1893. 28 Walsh T, Lee MK, Casadei S, et al. Detection of inherited mutations for breast and ovarian cancer using genomic capture and massively parallel sequencing. Proc Natl Acad Sci U $S$ A 2010; 107: 12629-33. 
29 Eisenhauer EA, Therasse P, Bogaerts J, et al. New response evaluation criteria in solid tumours: revised RECIST guideline (version 1.1). Eur J Cancer 2009; 45: 228-47.

30 Rustin GJ, Vergote I, Eisenhauer E, et al. Definitions for response and progression in ovarian cancer clinical trials incorporating RECIST 1.1 and CA 125 agreed by the Gynecological Cancer Intergroup (GCIG). Int J Gynecol Cancer 2011; 21: 419-23.

31 Brown EG, Wood L, Wood S. The medical dictionary for regulatory activities (MedDRA). Drug Saf 1999; 20: 109-17.

$32 \mathrm{NCl}$ Term Browser, CTCAE.

http://ctep.cancer.gov/protocolDevelopment/electronic_applications/ctc.htm (accessed June 16 2015).

33 Coleman RL, Swisher EM, Oza AM, et al. Refinement of prespecified cutoff for genomic loss of heterozygosity (LOH) in ARIEL2 part 1: A phase II study of rucaparib in patients (pts) with high grade ovarian carcinoma (HGOC). J Clin Oncol 2016; 34: 5540.

34 Liu JF, Barry WT, Birrer M, et al. Combination cediranib and olaparib versus olaparib alone for women with recurrent platinum-sensitive ovarian cancer: a randomised phase 2 study. The Lancet Oncology 2014; 15: 1207-14.

35 Birkbak NJ, Wang ZC, Kim JY, et al. Telomeric allelic imbalance indicates defective DNA repair and sensitivity to DNA-damaging agents. Cancer Discov 2012; 2: 366-75.

36 Popova T, Manié E, Rieunier G, et al. Ploidy and large-scale genomic instability consistently identify basal-like breast carcinomas with BRCA1/2 inactivation. Cancer Res 2012; 72: 5454-62.

37 Patch A-M, Christie EL, Etemadmoghadam D, et al. Whole-genome characterization of chemoresistant ovarian cancer. Nature 2015; 521: 489-94.

38 Kikuchi R, Lao Y, Bow DA, et al. Prediction of clinical drug-drug interactions of veliparib (ABT-888) with human renal transporters (OAT1, OAT3, OCT2, MATE1, and MATE2K). J Pharm Sci 2013; 102: 4426-32. 
39 Zhang Z-Z, Liu YJC, Yin X-L, Zhan P, Gu Y, Ni X-Z. Loss of BRCA1 expression leads to worse survival in patients with gastric carcinoma. World J Gastroenterol 2013; 19: 1968-74.

40 Isakoff SJ, Mayer EL, He L, et al. TBCRC009: a multicenter phase II clinical trial of platinum monotherapy with biomarker assessment in metastatic triple-negative breast cancer. $J$ Clin Oncol 2015; 33: 1902-9.

41 Robinson D, Van Allen Eliezer M, Wu Y-M, et al. Integrative clinical genomics of advanced prostate cancer. Cell 2015; 161: 1215-28. 
Table 1: Demographic and disease characteristics by HRD subgroup

\begin{tabular}{|c|c|c|c|c|}
\hline & & HRD subgrour & & \\
\hline Characteristic & $\begin{array}{l}B R C A \\
\text { mutant } \\
(n=40)\end{array}$ & $\begin{array}{c}\text { BRCA wild-type/ } \\
\text { LOH high } \\
(\mathrm{n}=82)\end{array}$ & $\begin{array}{c}\text { BRCA wild-type/ } \\
\text { LOH low } \\
(n=70)\end{array}$ & $\begin{array}{c}\text { Total } \\
(\mathrm{n}=204)^{\star}\end{array}$ \\
\hline Median age (range), years & $58 \cdot 5(33-78)$ & $65 \cdot 0(39-83)$ & $65 \cdot 0(31-86)$ & $64 \cdot 5(31-86)$ \\
\hline ECOG Performance Status, n (\%) & & & & \\
\hline 0 & $26(65 \cdot 0)$ & $52(63.4)$ & $46(65 \cdot 7)$ & $133(65 \cdot 2)$ \\
\hline 1 & $14(35 \cdot 0)$ & $30(36 \cdot 6)$ & $23(32 \cdot 9)$ & $70(34 \cdot 3)$ \\
\hline Pending & 0 & 0 & $1(1 \cdot 4)$ & $1(0 \cdot 5)$ \\
\hline Diagnosis, $\mathrm{n}(\%)^{\dagger}$ & & & & \\
\hline Epithelial ovarian cancer & $38(95 \cdot 0)$ & $68(82 \cdot 9)$ & $49(70 \cdot 0)$ & $163(79 \cdot 9)$ \\
\hline Primary peritoneal cancer & $1(2 \cdot 5)$ & $10(12 \cdot 2)$ & $12(17 \cdot 1)$ & $24(11 \cdot 8)$ \\
\hline Fallopian tube cancer & $1(2.5)$ & $4(4 \cdot 9)$ & $9(12 \cdot 9)$ & $16(7 \cdot 8)$ \\
\hline Histology, n (\%) & & & & \\
\hline Serous & $39(97.5)$ & $80(97 \cdot 6)$ & $66(94 \cdot 3)$ & $197(96 \cdot 6)$ \\
\hline Endometrioid & $1(2 \cdot 5)$ & $1(1 \cdot 2)$ & $2(2 \cdot 9)$ & $4(2 \cdot 0)$ \\
\hline Mixed & 0 & $1(1 \cdot 2)$ & $2(2 \cdot 9)$ & $3(1.5)$ \\
\hline Number of prior treatment regimens & & & & \\
\hline Median number of regimens (range) & $2 \cdot 0(1-6)$ & $1 \cdot 0(1-6)$ & $1 \cdot 0(1-3)$ & $1 \cdot 0(1-6)$ \\
\hline $1, \mathrm{n}(\%)$ & $17(42 \cdot 5)$ & $44(53 \cdot 7)$ & $47(67 \cdot 1)$ & $118(57 \cdot 8)$ \\
\hline$\geq 2, \mathrm{n}(\%)$ & $23(57 \cdot 5)$ & $38(46 \cdot 3)$ & $23(32 \cdot 9)$ & $86(42 \cdot 2)$ \\
\hline $\begin{array}{l}\text { Median number of platinum-based } \\
\text { regimens (range) }\end{array}$ & $2 \cdot 0(1-5)$ & $1 \cdot 0(1-5)$ & $1 \cdot 0(1-3)$ & $1 \cdot 0(1-5)$ \\
\hline $1, \mathrm{n}(\%)$ & $17(42 \cdot 5)$ & $45(54 \cdot 9)$ & $49(70 \cdot 0)$ & $121(59 \cdot 3)$ \\
\hline$\geq 2-$ no. (\%) & $23(57 \cdot 5)$ & $37(45 \cdot 1)$ & $21(30 \cdot 0)$ & $83(40 \cdot 7)$ \\
\hline $\begin{array}{l}\text { Progression-free interval following } \\
\text { completion of platinum-based }\end{array}$ & & & & \\
\hline
\end{tabular}


chemotherapy, n (\%)

6 to $<12$ months

$23(57 \cdot 5)$

$37(45 \cdot 1)$

$31(44 \cdot 3)$

$96(47 \cdot 1)$

$\geq 12$ months

$17(42 \cdot 5)$

$45(54 \cdot 9)$

$39(55 \cdot 7)$

$108(52 \cdot 9)$

ECOG=Eastern Cooperative Oncology Group. HRD=homologous recombination deficiency. LOH=loss of heterozygosity.

*Includes 12 patients (5.9\% of total) whose tumour specimens had sufficient nuclei to categorise as $B R C A$ wild-type, but insufficient nuclei to perform genomic LOH analysis.

${ }^{\dagger}$ Diagnosis was unknown for one patient $(0.5 \%$ of total). 
Table 2: Objective response rates by HRD subgroup

\begin{tabular}{|c|c|c|c|c|}
\hline \multirow[b]{3}{*}{ HRD subgroup } & \multicolumn{4}{|c|}{ Objective response rate } \\
\hline & \multicolumn{2}{|c|}{ By RECIST* } & \multicolumn{2}{|c|}{$\begin{array}{l}\text { By combined } \\
\text { RECIST/CA-125 }\end{array}$} \\
\hline & n (\%) & $95 \% \mathrm{Cl}$ & n (\%) & $95 \% \mathrm{Cl}$ \\
\hline$B R C A$ mutant $(\mathrm{n}=40)$ & $32(80 \cdot 0)$ & $64 \cdot 4-90 \cdot 9$ & $34(85 \cdot 0)$ & $70 \cdot 2-94 \cdot 3$ \\
\hline Germline mutation $(n=20)$ & $17(85 \cdot 0)$ & $62 \cdot 1-96 \cdot 8$ & $17(85 \cdot 0)$ & $62 \cdot 1-96 \cdot 8$ \\
\hline Somatic mutation $(n=19)$ & $14(73 \cdot 7)$ & $48 \cdot 8-90 \cdot 9$ & $16(84 \cdot 2)$ & $60 \cdot 4-96 \cdot 6$ \\
\hline Indeterminate $(\mathrm{n}=1)$ & $1(100 \cdot 0)$ & $2 \cdot 5-100 \cdot 0$ & $1(100 \cdot 0)$ & $2 \cdot 5-100 \cdot 0$ \\
\hline$B R C A 1$ mutation $(\mathrm{n}=29)$ & $23(79 \cdot 3)$ & $60 \cdot 3-92 \cdot 0$ & $25(86 \cdot 2)$ & $68 \cdot 3-96 \cdot 1$ \\
\hline BRCA2 mutation $(\mathrm{n}=11)$ & $9(81 \cdot 8)$ & $48 \cdot 2-97 \cdot 7$ & $9(81 \cdot 8)$ & $48 \cdot 2-97 \cdot 7$ \\
\hline PFI, $\geq 6$ to $<12$ months $(n=23)$ & $20(87 \cdot 0)$ & $66 \cdot 4-97 \cdot 2$ & $20(87 \cdot 0)$ & $66 \cdot 4-97 \cdot 2$ \\
\hline PFI, $\geq 12$ months $(n=17)$ & $12(70 \cdot 6)$ & $44 \cdot 0-89 \cdot 7$ & $14(82 \cdot 4)$ & $56 \cdot 6-96 \cdot 2$ \\
\hline$B R C A$ wild-type/LOH high $(\mathrm{n}=82)$ & $24(29 \cdot 3)$ & $19 \cdot 7-40 \cdot 4$ & $36(43.9)$ & $33 \cdot 0-55 \cdot 3$ \\
\hline$B R C A$ wild-type/LOH low $(\mathrm{n}=70)$ & $7(10 \cdot 0)$ & $4 \cdot 1-19 \cdot 5$ & $14(20 \cdot 0)$ & $11 \cdot 4-31 \cdot 3$ \\
\hline$B R C A$ wild-type/LOH not classified $(\mathrm{n}=12)$ & $4(33 \cdot 3)$ & $9 \cdot 9-65 \cdot 1$ & $7(58 \cdot 3)$ & $27 \cdot 7-84 \cdot 8$ \\
\hline
\end{tabular}

CA-125=cancer antigen 125. $\mathrm{Cl}=$ confidence interval. $\mathrm{HRD}=$ homologous recombination deficiency.

$\mathrm{LOH}=$ loss of heterozygosity. $\mathrm{PFI}=$ progression-free interval following completion of platinum-based chemotherapy. RECIST=Response Evaluation Criteria In Solid Tumors version 1·1.

Confidence intervals calculated using Clopper-Pearson methodology.

${ }^{*}$ Confirmed responses according to RECIST. 
Table 3: Treatment-emergent adverse events

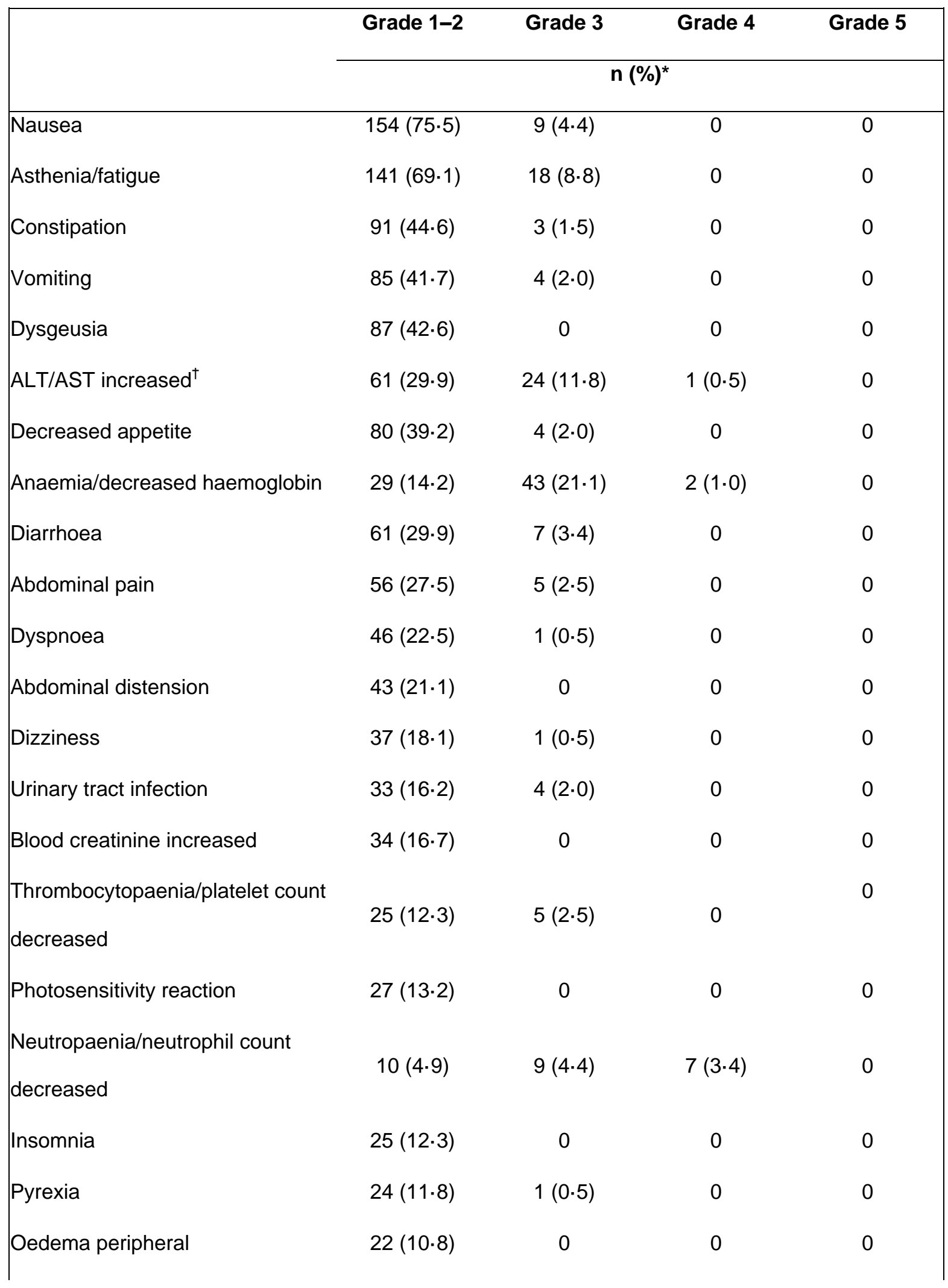




\begin{tabular}{|c|c|c|c|c|}
\hline Alopecia & $21(10 \cdot 3)$ & 0 & 0 & 0 \\
\hline Stomatitis & $20(9 \cdot 8)$ & $1(0.5)$ & 0 & 0 \\
\hline Upper respiratory tract infection & $21(10 \cdot 3)$ & 0 & 0 & 0 \\
\hline $\begin{array}{l}\text { Blood alkaline phosphatase } \\
\text { increased }\end{array}$ & $16(7 \cdot 8)$ & $3(1.5)$ & 0 & 0 \\
\hline Dyspepsia & $18(8 \cdot 8)$ & $1(0 \cdot 5)$ & 0 & 0 \\
\hline Pain in extremity & $17(8 \cdot 3)$ & $1(0.5)$ & 0 & 0 \\
\hline Weight decreased & $16(7 \cdot 8)$ & $1(0 \cdot 5)$ & 0 & 0 \\
\hline Dehydration & $10(4 \cdot 9)$ & $6(2 \cdot 9)$ & 0 & 0 \\
\hline Myalgia & $15(7 \cdot 4)$ & $1(0.5)$ & 0 & 0 \\
\hline Ascites & $7(3 \cdot 4)$ & $7(3 \cdot 4)$ & 0 & 0 \\
\hline Blood cholesterol increased & $11(5 \cdot 4)$ & $2(1 \cdot 0)$ & 0 & 0 \\
\hline Hypokalaemia & $7(3.4)$ & $5(2 \cdot 5)$ & 0 & 0 \\
\hline White blood cell count decreased & $11(5 \cdot 4)$ & $1(0.5)$ & 0 & 0 \\
\hline Small intestinal obstruction & $1(0.5)$ & $10(4 \cdot 9)$ & 0 & 0 \\
\hline Hydronephrosis & $8(3.9)$ & $2(1 \cdot 0)$ & 0 & 0 \\
\hline Malignant neoplasm progression & 0 & $8(3.9)$ & $1(0.5)$ & $1(0 \cdot 5)$ \\
\hline Blood bilirubin increased & $7(3.4)$ & $1(0.5)$ & $1(0.5)$ & 0 \\
\hline Mucosal inflammation & $7(3.4)$ & $1(0.5)$ & 0 & 0 \\
\hline Acute kidney injury & $1(0.5)$ & $5(2 \cdot 5)$ & 0 & 0 \\
\hline Bronchitis & $5(2 \cdot 5)$ & $1(0.5)$ & 0 & 0 \\
\hline $\begin{array}{l}\text { Gamma-glutamyltransferase } \\
\text { increased }\end{array}$ & $1(0.5)$ & $4(2 \cdot 0)$ & $1(0.5)$ & 0 \\
\hline Hypercholesterolaemia & $5(2 \cdot 5)$ & $1(0.5)$ & 0 & 0 \\
\hline Hyperglycaemia & $4(2 \cdot 0)$ & $2(1 \cdot 0)$ & 0 & 0 \\
\hline Rectal haemorrhage & $5(2.5)$ & $1(0.5)$ & 0 & 0 \\
\hline
\end{tabular}




\begin{tabular}{|c|c|c|c|c|}
\hline Fall & $4(2 \cdot 0)$ & $1(0 \cdot 5)$ & 0 & 0 \\
\hline Transaminases increased & $2(1 \cdot 0)$ & $3(1.5)$ & 0 & 0 \\
\hline Malaise & $3(1 \cdot 5)$ & $1(0 \cdot 5)$ & 0 & 0 \\
\hline Sepsis & 0 & $1(0 \cdot 5)$ & $2(1 \cdot 0)$ & $1(0 \cdot 5)$ \\
\hline Presyncope & $2(1 \cdot 0)$ & $1(0 \cdot 5)$ & 0 & 0 \\
\hline Pulmonary embolism & $1(0 \cdot 5)$ & $2(1 \cdot 0)$ & 0 & 0 \\
\hline Syncope & 0 & $3(1.5)$ & 0 & 0 \\
\hline Food poisoning & $1(0 \cdot 5)$ & $1(0 \cdot 5)$ & 0 & 0 \\
\hline Hyperbilirubinaemia & $1(0 \cdot 5)$ & $1(0 \cdot 5)$ & 0 & 0 \\
\hline Lymphocyte count decreased & $1(0 \cdot 5)$ & 0 & $1(0 \cdot 5)$ & 0 \\
\hline Lymphoedema & $1(0 \cdot 5)$ & $1(0 \cdot 5)$ & 0 & 0 \\
\hline Tachycardia & $1(0 \cdot 5)$ & $1(0 \cdot 5)$ & 0 & 0 \\
\hline Pneumonia & 0 & $2(1 \cdot 0)$ & 0 & 0 \\
\hline Agitation & 0 & $1(0 \cdot 5)$ & 0 & 0 \\
\hline Bile duct obstruction & 0 & $1(0.5)$ & 0 & 0 \\
\hline Dyspareunia & 0 & $1(0.5)$ & 0 & 0 \\
\hline Empyema & 0 & $1(0 \cdot 5)$ & 0 & 0 \\
\hline Hypermagnesaemia & 0 & $1(0 \cdot 5)$ & 0 & 0 \\
\hline Intestinal obstruction & 0 & $1(0 \cdot 5)$ & 0 & 0 \\
\hline Lymphangitis & 0 & $1(0.5)$ & 0 & 0 \\
\hline Mental status changes & 0 & $1(0.5)$ & 0 & 0 \\
\hline Peritonitis & 0 & $1(0.5)$ & 0 & 0 \\
\hline Cataract & 0 & 0 & $1(0 \cdot 5)$ & 0 \\
\hline Febrile neutropenia & 0 & 0 & $1(0.5)$ & 0 \\
\hline Granulocyte count decreased & 0 & 0 & $1(0 \cdot 5)$ & 0 \\
\hline Intestinal perforation & 0 & 0 & $1(0.5)$ & 0 \\
\hline
\end{tabular}




\begin{tabular}{lcccc|} 
Large intestinal obstruction & 0 & 0 & $1(0.5)$ & 0 \\
Long QT syndrome congenital & 0 & 0 & $1(0.5)$ & 0 \\
AML/MDS & 0 & 0 & 0 & 0 \\
\hline
\end{tabular}

$\mathrm{ALT}=$ alanine aminotransferase. $\mathrm{AML}=$ acute myeloid leukaemia. $\mathrm{AST}=$ aspartate aminotransferase.

MDS=myelodysplastic syndrome.

${ }^{*} \mathrm{n}=204$.

${ }^{\dagger}$ ALT/AST elevations were transient, self-limiting, and not associated with other signs of liver toxicity. 


\section{Figure legends}

\section{Figure 1: Diagram of patient subgroups}

$\mathrm{LOH}=$ loss of heterozygosity.

*Data presented in this manuscript focus on those patients whose LOH status was determined.

${ }^{\dagger}$ Patients had genomic $\mathrm{LOH} \geq 14 \%$.

${ }^{\ddagger}$ Sequencing of archival and pretreatment tumour samples from one patient did not pass quality check; therefore, the tumour cannot be definitively concluded to be BRCA wild-type.

\section{Figure 2: Progression-free survival and duration of response by HRD subgroup}

(A) Kaplan-Meier estimates of progression-free survival in all patients who received at least one dose of rucaparib, stratified by HRD subgroup. Median progression-free survival for the $B R C A$ mutant (blue), BRCA-wild-type/LOH-high (red), and BRCA-wild-type/LOH-low (green) subgroups was $12 \cdot 8(95 \% \mathrm{Cl}, 9 \cdot 0-14 \cdot 7), 5 \cdot 7(95 \% \mathrm{Cl}, 5 \cdot 3-7 \cdot 6)$, and $5 \cdot 2(95 \% \mathrm{Cl}, 3 \cdot 6-5 \cdot 5)$ months. (B) Swimlane plot of duration of response in patients classified into the three predefined HRD subgroups with confirmed partial or complete RECIST responses. Each bar represents an individual patient with the length corresponding to length of time on study drug. Duration of a response is indicated by shading along each bar. Tiles to the left of the plot indicate the HRD subgroup of each patient and homologous recombination gene mutation type (colour coded by type) or methylation type identified in tumour or blood samples. Germline mutations are indicated by a "G," somatic mutations by an "S," and indeterminate mutations by an "I." Patients with unknown methylation of BRCA1 or RAD51C are indicated by an "X." One patient with a $B R C A 1$ methylation who had a partial response is not included in panel $\mathrm{B}$ because the archival or pretreatment biopsy could not be classified into an HRD subgroup. 
$\mathrm{Cl}=$ confidence interval. $\mathrm{HR}=$ hazard ratio. $\mathrm{HRD}=$ homologous recombination deficiency. $\mathrm{LOH}=$ loss of heterozygosity.

\section{Figure 3: Best response in size of target lesions by HRD subgroup}

Best percentage change from baseline in sum of longest diameter of target lesions according to RECIST is shown in the (A) BRCA-mutant subgroup, (B) BRCA-wild-type/LOH-high subgroup, and (C) BRCA-wild-type/LOH-low subgroup. Each bar represents percentage change from baseline in sum of the longest diameter of target lesions for an individual patient according to RECIST. Upper dotted line indicates the threshold for progressive disease, a $20 \%$ increase in the sum of the longest diameter of the target lesions. Lower dotted line indicates the threshold for partial response, a $30 \%$ decrease in the sum of the longest diameter of the target lesions. Patients who also had a CA-125 response are indicated by cross-hatched bars. Ongoing patients at the time of the data cutoff are indicated by a " $₫$ " symbol. The tables below each panel indicate homologous recombination gene mutations (colour coded by type) and methylation identified in the tumour samples. Germline mutations are indicated by a "G," somatic mutations by an "S," and indeterminate mutations by an "I." Patients with unknown methylation of $B R C A 1$ or RAD51C are indicated by an "X."

CA-125=cancer antigen 125. HRD=homologous recombination deficiency. $\mathrm{LOH}=$ loss of heterozygosity. RECIST=Response Evaluation Criteria In Solid Tumors version 1·1. 


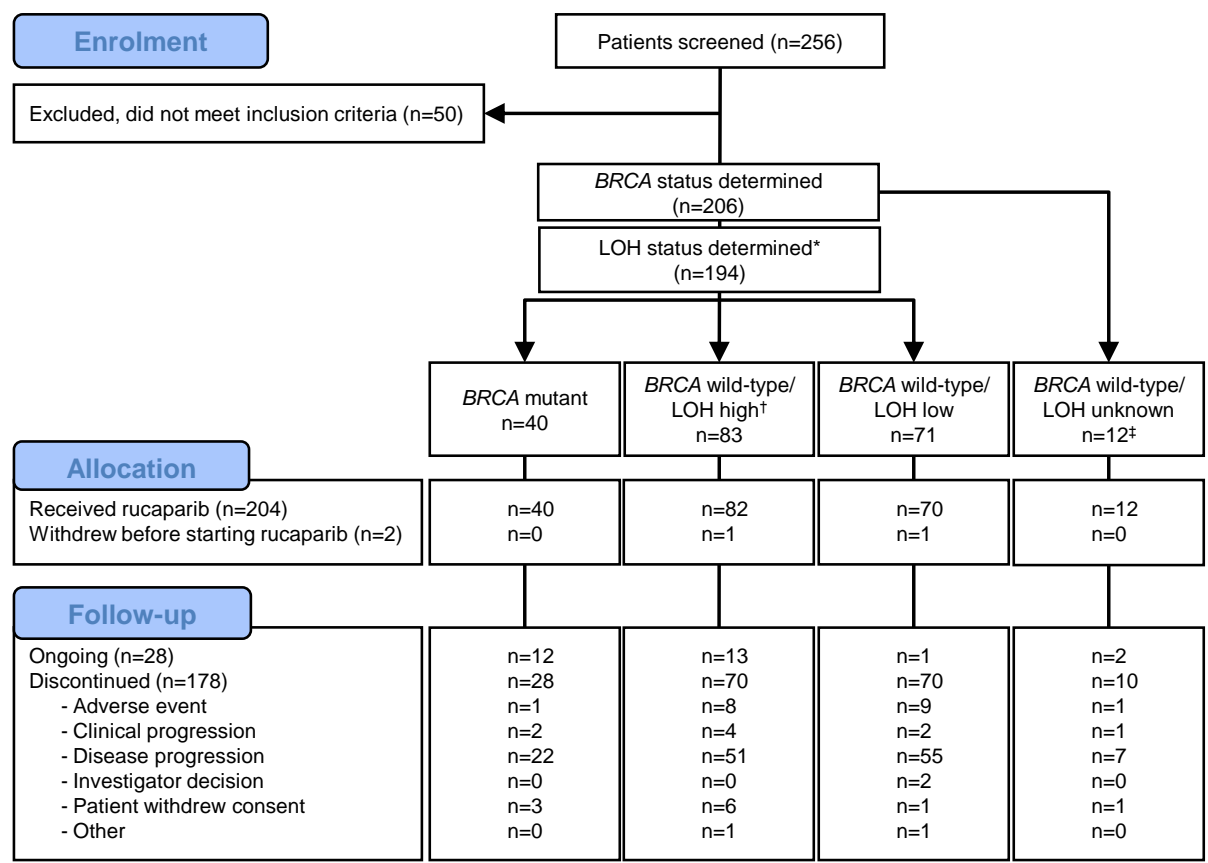

$\mathrm{LOH}=$ loss of heterozygosity.

${ }^{*}$ Data presented in this manuscript focus on those patients whose LOH status was determined.

†Patients had genomic $\mathrm{LOH} \geq 14 \%$.

‡Sequencing of archival and pretreatment tumour samples from one patient did not pass quality check; therefore, the tumour cannot be definitively concluded to be BRCA wild-type. 
Figure 2

A

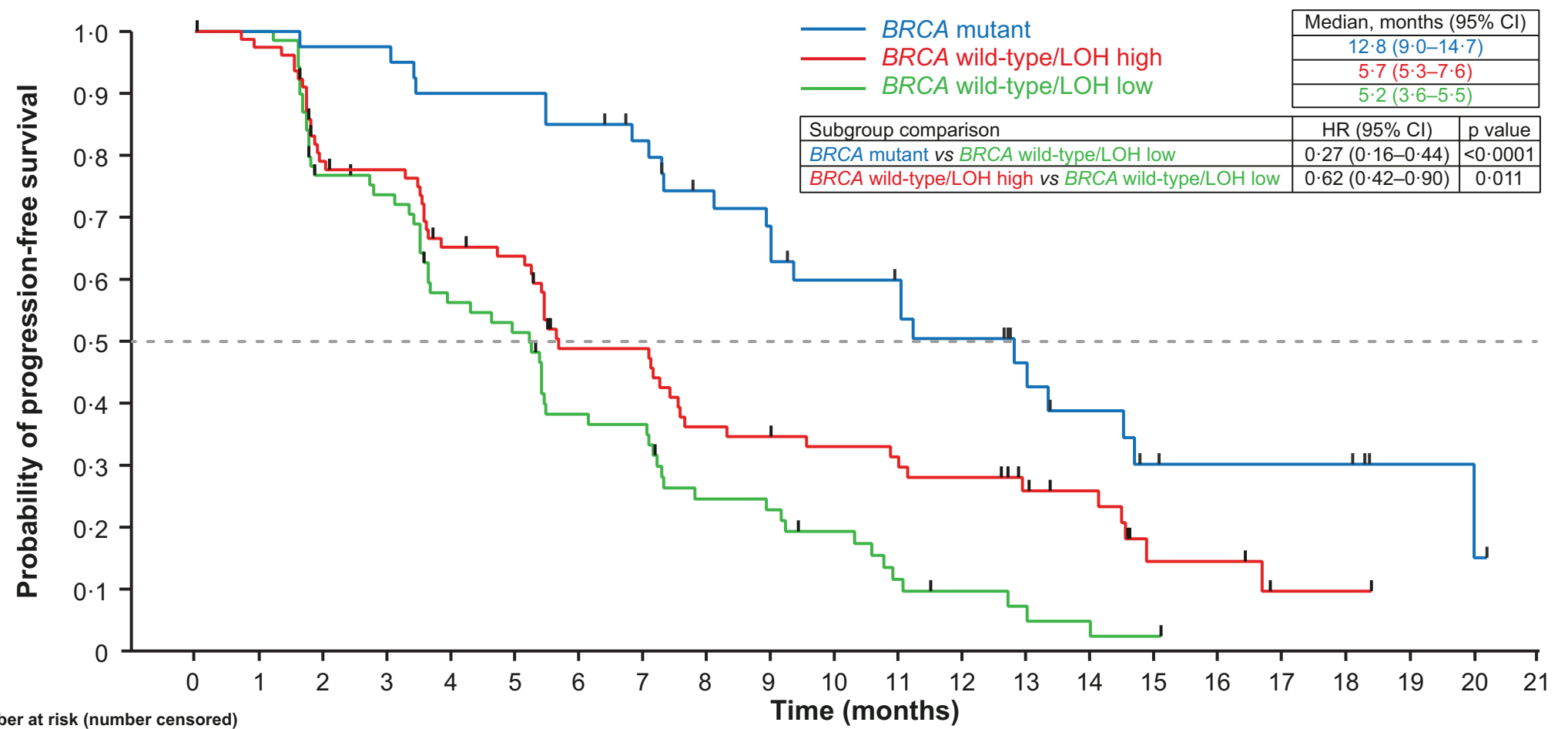

Number at risk (number censored)

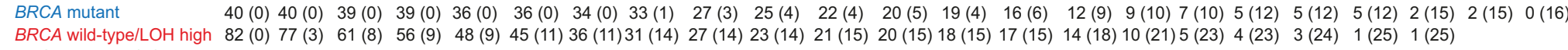
BRCA wild-type/LOH low $70(0) 69(1) \quad 53(2) \quad 48(5) \quad 37(5) \quad 34(6) \quad 23(7) \quad 22(7) \quad 15(8) \quad 14(8) \quad 12(8) \quad 10(9) \quad 6(9) \quad 4(10) \quad 3(10) \quad 2(10) \quad 1(10) \quad 0(11)$

B

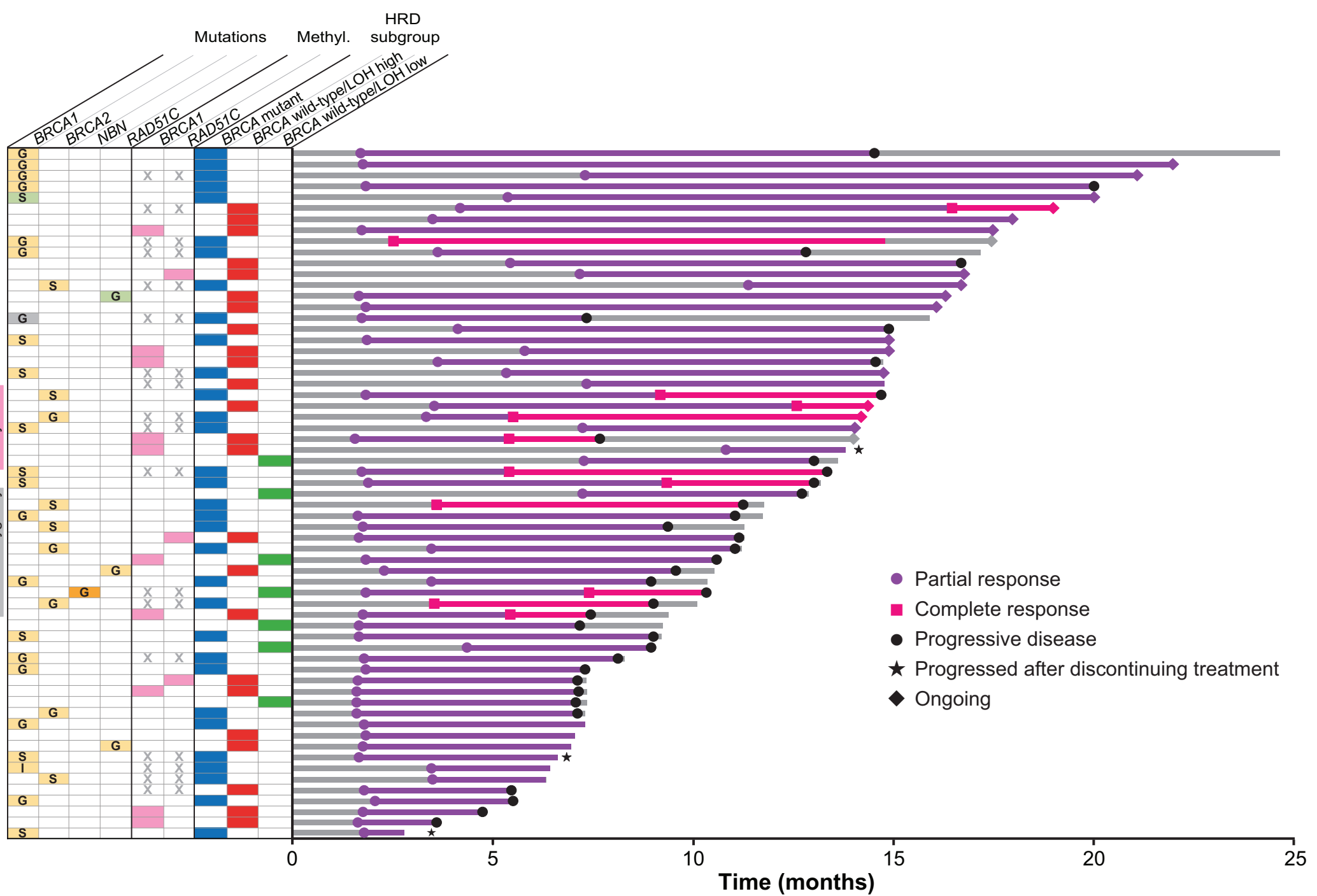


Figure 3

A

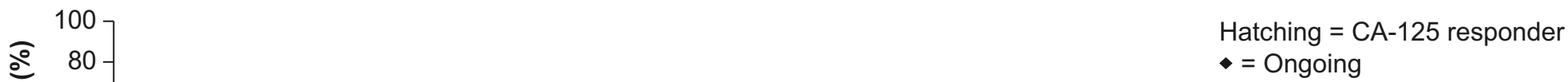

- = Ongoing
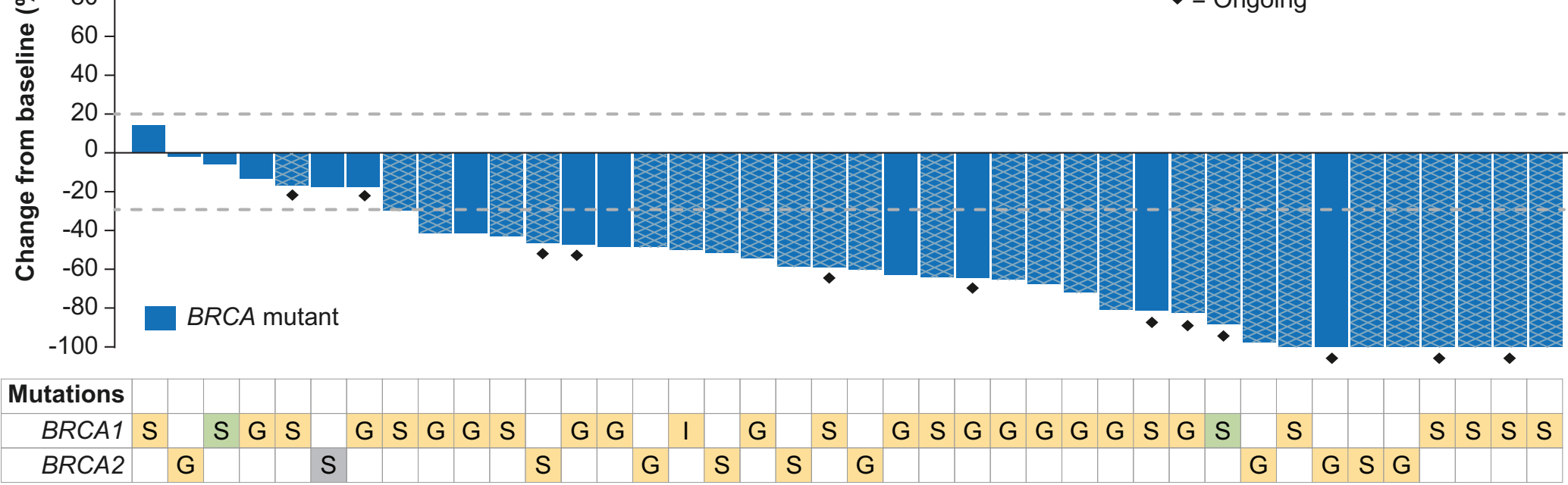

B

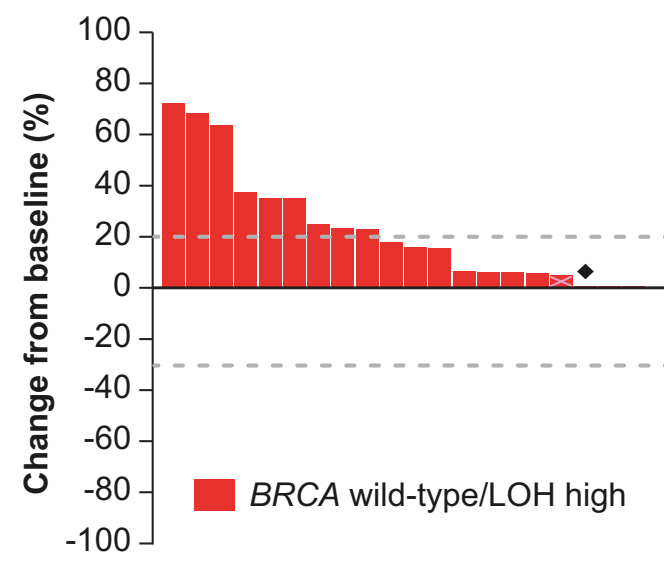

Hatching = CA-125 responder

- = Ongoing
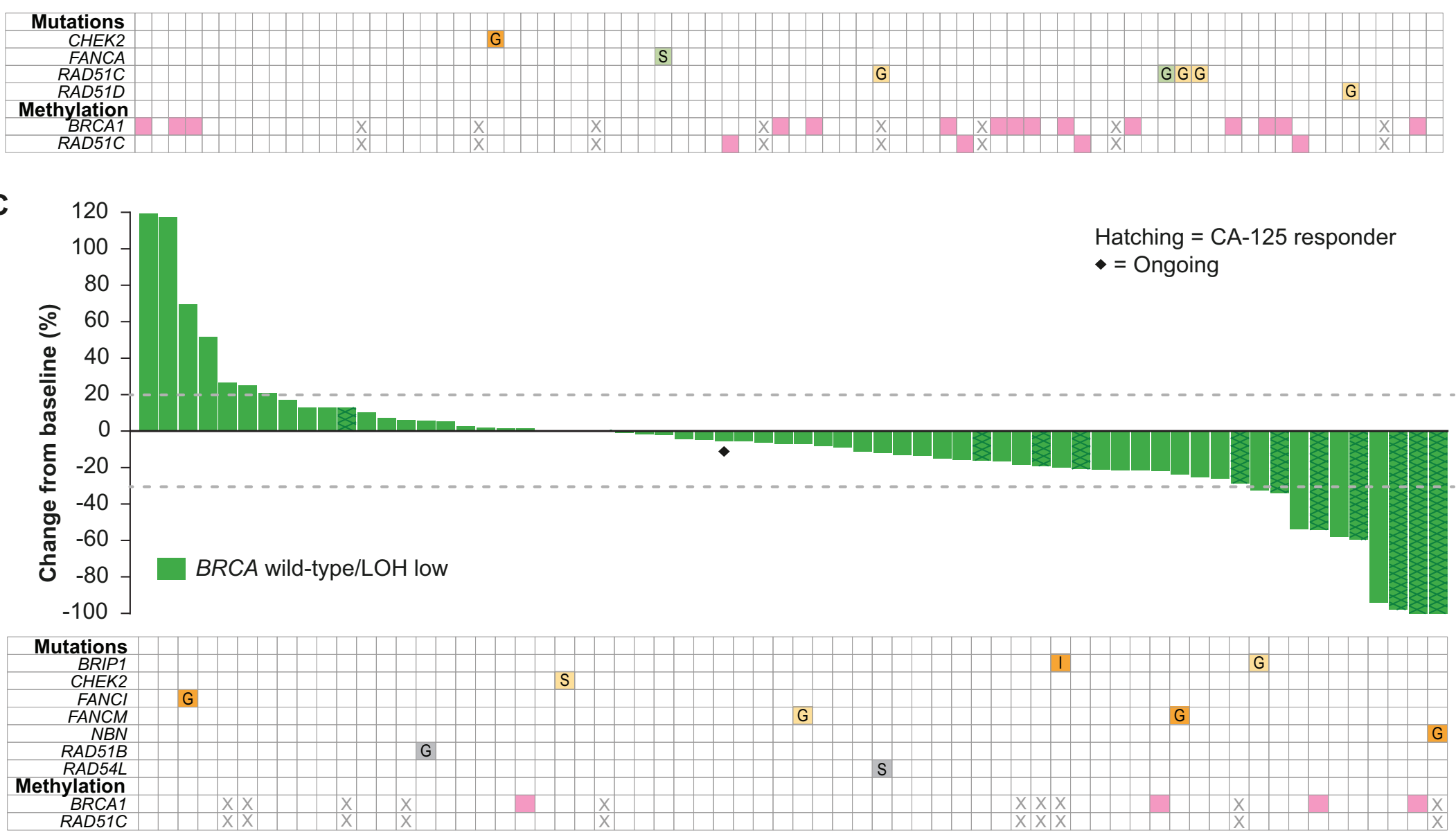